\title{
The Charge Structure of the Nucleons
}

\author{
M. Y. Hussein \\ Department of Physics, University of Bahrain, Bahrain \\ Email:myhussain@uob.edu.bh
}

How to cite this paper: Hussein, M.Y. (2017) The Charge Structure of the Nucleons. Journal of Modern Physics, 8, 1933-1937. https://doi.org/10.4236/jmp.2017.812115

Received: October 10, 2017

Accepted: November 7, 2017

Published: November 10, 2017

Copyright (C) 2017 by author and Scientific Research Publishing Inc. This work is licensed under the Creative Commons Attribution International License (CC BY 4.0).

http://creativecommons.org/licenses/by/4.0/

\begin{abstract}
Study of nucleons charge radii and electromagnetic form factors are expected to provide valuable information about the distribution of electric charge within the fundamental particles in nucleon's inner structure. In the recent years, dramatic progress has been made in the understanding of the nucleon structure and the precision of its partonic content, due to the vast theoretical progress, and the availability of new high precision measurements. Here in this article, we present a simple model for the charge structure of the nucleons and the most available sets of the structure functions to calculate the mean square charge radius $\left\langle r_{N}^{2}\right\rangle$ for both protons and neutrons. Our results are consistent with the modern understanding of the nucleons as well as recent experimental data. We discuss the origin of the sign $\left\langle r_{N}^{2}\right\rangle$ for both proton and neutron.
\end{abstract}

\section{Keywords}

Nucleon Charge Structure, Electromagnetic Form Factors, Quark-Parton Model, Charge Radius

\section{Introduction}

Nucleons charge structure has been a subject of extensive investigation for the past years [1]-[7]. The electromagnetic form factors and charge radii of nucleons provide critical information about the distribution of the charge within the nucleons and reveal high valuable information about the dynamic of the strong interaction. The non-uniform charge distribution leads to a finite value for the mean square charge radii of nucleons.

There have been several attempts to extract more information on inner nucleon structure. Variety of techniques are used in order to calculate nucleons mean square charge radius $\left\langle r_{N}^{2}\right\rangle$ and the electromagnetic form factors including, the empirical charge distribution, the quark-parton model and composite 
models of the hadrons have achieved considerable popularity, primarily because the most elementary, non-relativistic quark picture accounts remarkably well for most of the available data on hadronic physics.

Recently, the question of that the mean square intrinsic charge radius of the nucleons is widely discussed and has been elegantly explained in terms of generalized parton distributions and the charge density related to the electric charge distribution $\rho(r)$ inside the nucleon [8], which has been given by

$$
\left\langle r_{N}^{2}\right\rangle=\int \rho(r) r^{2} \mathrm{~d}^{3} r
$$

The non-zero value of the charge radius of the nucleon implies a non-vanishing electric form factors since

$$
\left\langle r_{N}^{2}\right\rangle=-\left.6 \frac{\mathrm{d} G_{E}^{N}\left(q^{2}\right)}{\mathrm{d} q^{2}}\right|_{q^{2}=0}
$$

where $G_{E}^{N}\left(q^{2}\right)$ is the electromagnetic form factors. The most recent determination of the thermal neutron-electron interaction predicts a value of [9]

$$
\left.\frac{\mathrm{d} G_{E}^{N}\left(q^{2}\right)}{\mathrm{d} q^{2}}\right|_{q^{2}=0}=0.02 \pm 0.0014 \mathrm{fm}^{2}
$$

where $G_{E}^{N}\left(q^{2}\right)$ is the electromagnetic form factors. Earlier electron-deuteron and electron-proton scattering data has been analyzed very precisely in $q^{2}$. The low $q^{2}$ part of the data has been used to extract the slope of $G_{E}^{n}$, the value obtained was

$$
\left.\frac{\mathrm{d} G_{E}^{n}\left(q^{2}\right)}{\mathrm{d} q^{2}}\right|_{q^{2}}=0.02 \pm 0.0014 \mathrm{fm}^{2}
$$

where $G_{E}^{N}\left(q^{2}\right)$ is the electromagnetic form factors. Equation (3) and Equation (4) show essential agreement between both low $q^{2}$ scattering electron proton and electron Deuteron with data from thermal neutron-electron experiment. The neutron charge radius is far from small on the scale of proton. The available data [9] imply that

$$
\frac{\left\langle r_{n}^{2}\right\rangle}{\left\langle r_{p}^{2}\right\rangle} \cong-0.155
$$

where $\left\langle r_{p}^{2}\right\rangle$ and $\left\langle r_{n}^{2}\right\rangle$ is the corresponding proton and neutron mean square charge radius.

\section{Nucleon Charge Structure}

In the context of the proton model, a connection has been established by Sehgal [10] between the sign of the neutron charge radius and the distribution of proton transverse momenta. Moreover, a contradiction between the observed negative sign and the trend of deep inelastic data was noted in ref. [11]. Calculation in ref. [11] [12] related the negativeness of $\left\langle r_{n}^{2}\right\rangle$ to the nucleons possible 
quark-diquark structure. The effect of nucleons quark-diquark structure is added to the usual constituent quark model to calculate nucleons charge square radius. All results of $\left\langle r_{p}^{2}\right\rangle$ and $\left\langle r_{n}^{2}\right\rangle$ are fitted to the experimental value extremely well.

In this work we try to relate the negative value of $\left\langle r_{n}^{2}\right\rangle$ to the distribution of parton transverse momentum with the general prescriptions of quark-parton model. Our consideration is that the nucleon can be described in the parton model by a set of functions regarded as an integral over a transverse and longitudinal momentum distribution as:

$$
f_{i}(x)=\int h_{i}(x, b) \mathrm{d} b
$$

With, $h_{i}(x, b)=[\pi A(x)] f_{i}(x) \exp \left[-b^{2} / A(x)\right], \quad f_{i}(y)=\int_{0}^{z_{o}} g_{i}(y, z) \mathrm{d} z$ and $g_{i}(y, z)=\left[z_{o}\left(1-\frac{z_{o}^{2}}{3 B(y)}\right)\right]^{-1} f_{i}(y)\left(1-\frac{z^{2}}{B(y)}\right)$,

where $z_{o}$ is an appropriate cutoff related to the thickness of the nucleon. Our considerations are based on the hypothesis that the nucleon has a disc-like look instead of a spherical object on account of the relativistic contraction as suggested in ref. [12], which characterized by Equation (6). In this simple model of the nucleon, the complete quark-parton distribution function can be written in a separable form as

$$
\rho_{i}(x, b ; y, z)=h_{i}(x, b) g_{i}(y, z)
$$

Expression [1] for the mean square charge radius can be rewritten in the form

$$
\left\langle r^{2}\right\rangle=\iint \mathrm{d} x \mathrm{~d} y \delta\left(x^{2}+y^{2}-1\right) \sum e_{i} \int \mathrm{d}^{3} r r^{2} \rho(r)
$$

With $x^{2}+y^{2}=1, \mathrm{~d}^{3} r=\mathrm{d}^{2} b \mathrm{~d} z, r^{2}=b^{2}+z^{2}$.

Integrating Equation (8) over $r$ and $y$, the resulting expression explicitly gives

$$
\left\langle r^{2}\right\rangle=\int_{0}^{1} \mathrm{~d} x[A(x)+\eta(y)] \frac{L(x)}{2 y}
$$

Having $A(x)=\exp (-\beta x), \quad \eta(y)=\frac{z_{0}^{2}}{5}\left(\frac{5 A(y)-3 z_{0}^{2}}{3 A(y)-z_{0}^{2}}\right)$.

$L(x)$ represents the compound quark density function for the nucleon

$$
\begin{aligned}
& L_{p}(x)=\frac{2}{3} u_{v}(x) u_{v}(y)-\frac{1}{3} d_{v}(x) d_{v}(y) \\
& L_{n}(x)=\frac{2}{3} d_{v}(x) d_{v}(y)-\frac{1}{3} u_{v}(x) u_{v}(y)
\end{aligned}
$$

where $u_{v}(x)$ and $d_{v}(x)$ are the parton distribution function as extracted from global fits to a wide range of deep-inelastic and other related data [13] [14]. The $v$ subscript refers to the valance constituency only. The form of $A(x)$ in Equation (9) is not unique and parameterized with $\beta$ which is itself a function of the scale $Q$ in $\mathrm{GeV}$. The form of $A(x)$ does not have to be the same for all nucleons. For $u_{v}$ and $d_{v}$ we make use of the structure distribution function from MSTW [13] and CTEQ [14]. 


\section{Results}

Equation (9) depends on the disc model itself and hence the simplicity of this treatment allows us to point out the following:

1) By choosing an appropriate value for $\beta$ we can reproduce the current signs and values for both $\left\langle r_{n}^{2}\right\rangle$ and $\left\langle r_{p}^{2}\right\rangle$ as shown in Table 1.

2) Because $u_{v}$ is significantly larger than $d_{v}$ in $\mathrm{x}$-space, and considering Equation (10), the calculation of $\left\langle r^{2}\right\rangle$ is dominated by $u_{v}$ such that Equation (10) can be well approximated by

$$
\begin{aligned}
& L_{p}(x) \cong \frac{2}{3} u_{v}(x) u_{v}(y) \\
& L_{n}(x) \cong-\frac{1}{3} u_{v}(x) u_{v}(y)
\end{aligned}
$$

This clearly shows that $\left\langle r_{p}^{2}\right\rangle>0$, while $\left\langle r_{n}^{2}\right\rangle<0$.

In fact, for the same $\beta$ one obtains a factor of -0.15 for the ratio in Equation (5). Thus even with the arbitrariness of choosing $\beta$ the signs of $\left\langle r^{2}\right\rangle$ are well understood in this treatment which suggest that the u-quark is the dominating factor in the nucleon charge distribution.

3) The uncertainty associated with the choice of parton distribution function do not affect our conclusions as we have used different sets and obtained similar results. In fact usually a limited shift in $\beta$ is all it took to reproduce the same results with a different set of parton distribution functions.

\section{Conclusion}

We have used a simple model of the nucleon in connection with the quark-parton model and the current information on parton distributions to re-establish both the value and the sign of the nucleon charge radii. It seems that the up quark is dominating the calculations of the root mean square charge radii. Equations (11a) and (11b) shows clearly why positive sign appear in mean square charge radius for the proton and why negative sign appear for the neutron. The major uncertainty in this work is the fact that the parton distributions

Table 1. Results reproduce the current signs and values for both $\left\langle r_{n}^{2}\right\rangle$ and $\left\langle r_{p}^{2}\right\rangle$.

\begin{tabular}{ccc}
\hline A) With $\left\langle r_{p}^{2}\right\rangle$ fixed at $0.707 \mathrm{fm}^{2}$ & \\
\hline Parton distribution & $\beta$ & $\left\langle r_{n}^{2}\right\rangle$ in $f m^{2}$ \\
\hline MRS T & 14.15 & -0.378 \\
CTEQ & 9.13 & -0.376 \\
\hline B) Independent $\left\langle r_{p}^{2}\right\rangle$ & & \\
\hline Parton distribution & $\beta$ & -0.120 \\
MRS T & 12.72 & -0.120 \\
\hline CTEQ & 7.70 &
\end{tabular}


used for both the neutron and the proton are those extracted from proton data only.

\section{References}

[1] Kohl, M. (2008) Nuclear Physics A, 805, 361.

[2] Nikolenko V.G. and Popov, A.B. (1992) Z. Phys, A, 341, 465.

[3] Alexandrov, Y.A. (1992) Z. Phys, A, 344, 219.

[4] Kopecki, S., Harvey, J.V., Nill, N., Riebs, P. and Schmidynayer, J. (1992) Baryon 92 International Conference on the Structure of Baryons and Related Meson, Yale Univ, New Haven.

[5] Alexandrov, Y.A. (1994) Report No JINR E3-92-384.

[6] Alexandrov, Y.A. (1992) Fundamental Properties of the Neutron. Oxford.

[7] Leeb, H. and Teichtmeister, C. (1993) Physical Review C, 48, 1719.

[8] Gentile, T.R. and Crawford, C.B. (2011) Physical Review C, 83, 055203.

[9] Tzeng, Y. and Hsiao, S. (1993) Nuovo Cimento, 106A, 573.

[10] Sehgal, L.M. (1974) Physics Letters, 53B, 106.

[11] Neigawa, A. and Kiang, D. (1976) Physical Review D, 14, 3235.

[12] Parashar, D. and Kaushal, R.S. (1976) Physical Review D, 13, 2684.

[13] Martin, A.D., Stirling, W.J., Thorne, R. and Watt, G. (2009) The European Physical Journal, C64, 653.

[14] Pumplin, J., et al. (2006) JHEP 0602, 032. 